\title{
Aplicação de redes Neuro Fuzzy ao processamento de peças automotivas por meio de injeção de polímeros
}

\author{
Carlos de Oliveira Affonso ${ }^{a}$, Renato José Sassi ${ }^{\text {b* }}$ \\ aaffonso.unesp@gmail.com, UNESP, Brasil \\ b*rjsassi@gmail.com, Poli-USP e UNINOVE, Brasil
}

\begin{abstract}
Resumo
0 processamento de peças automotivas por meio de injeção de polímeros envolve vários fenômenos físicos que ocorrem simultaneamente e que possuem caráter não linear e multivariável. Softwares comerciais podem ser utilizados na previsão dos parâmetros do processo, o que pode ser caro e inviável. Pode-se determinar os parâmetros de forma analítica, mas o tratamento desse problema requer a aplicação de teorias clássicas dos fenômenos de transporte, de difícil equacionamento. As redes NeuroFuzzy são aplicáveis a esse problema porque reúnem a capacidade de aprender das redes neurais artificiais com a capacidade da lógica Fuzzy de transformar variáveis linguísticas em regras. Neste trabalho combinou-se uma rede neural artificial Multilayer Perceptron e uma rede neural artificial Radial Basis Function à lógica Fuzzy para construir-se um modelo de inferência que previu o tempo de ciclo de processos de injeção de polímeros. Os resultados obtidos confirmam as redes NeuroFuzzy como opção para esse tipo de problema.

Palavras-chave

Indústria automotiva. Polímeros. Processo de injeção. Redes NeuroFuzzy.
\end{abstract}

\section{Introdução}

Devido a sua fácil processabilidade, grande versatilidade e ao seu baixo custo de produção, principalmente quando utilizado em grandes volumes, os materiais poliméricos vêm sendo utilizados em larga escala na indústria automotiva (Glauch et al., 1997; He et al., 2001; Canevarollo, 2002; Hemais, 2003; Bissoto \& Sydenstricker, 2006; Guarnieri \& Hatakeyama, 2010; Amatucci \& Bernardes, 2009; Salerno et al., 2010; Ozturk et al., 2012).

0 processamento de peças através de injeção de polímeros (plásticos) é complexo, uma vez que envolve vários fenômenos físicos que ocorrem simultaneamente, os quais possuem caráter não linear e estocástico (Yamaguchi, 2008; Cao et al., 2011).

Para que os critérios de custo e qualidade sejam atendidos o especialista humano deve intervir ajustando os parâmetros envolvidos. Dessa forma, pode-se afirmar que o processamento de polímeros possui as seguintes características: complexo, de caráter multivariável, incerto quanto à obtenção e controle das variáveis, dependente do conhecimento de especialistas.
Existem vários softwares comerciais que utilizam ferramentas de modelação via elementos finitos, para previsão dos parâmetros desse processo, previsões estas baseadas nas propriedades reológicas, térmicas, e exata geometria do produto, mas o software escolhido para tal atividade pode ser caro e por isso inviável.

Outra forma de determinar esses parâmetros pode ser feita de forma analítica, mas o tratamento desse problema através da aplicação das teorias clássicas dos fenômenos de transporte via equações de NavierStokes requer um difícil equacionamento que depende de características precisas da máquina injetora, da geometria do produto e dos parâmetros do processo (Agassant et al., 1991).

Para esse tipo de aplicação a lógica Fuzzy é recomendada, uma vez que as fronteiras do problema são incertas e o seu comportamento é não linear (Zadeh, 1965). A lógica Fuzzy fornece ferramental matemático para tratar as incertezas, ambiguidades e divagações associadas ao processo cognitivo e utilizá-lo 
para criar um modelo de inferência (Medeiros et al., 2007; Aguiar \& Salomon, 2007; Correia et al., 2011).

Neste trabalho, a lógica Fuzzy foi associada às redes neurais artificiais (RNAs), que inspiradas no modo como os neurônios do cérebro humano se conectam e transmitem a informação podem aprender por meio de exemplos (Kovács, 1996). Essas redes são compostas por estruturas simples de processamento conhecidas como neurônios artificiais (Haykin, 2001).

As RNAs possuem uma ampla área de aplicação como previsão de risco de crédito (Selau \& Ribeiro, 2009; Steiner et al., 2007), medicina (Blazadonakis \& Michalis, 2008) e em polímeros (Contant et al., 2004), entre outras.

Segundo Goldschmidt \& Passos (2005), técnicas podem ser combinadas para gerar as chamadas Arquiteturas Híbridas. A grande vantagem desse tipo de sistema deve-se ao sinergismo obtido pela combinação de duas ou mais técnicas. Esse sinergismo reflete na obtenção de um sistema mais poderoso (em termos de interpretação, de aprendizado, de estimativa de parâmetros, de generalização, dentre outros) e com menos deficiências. Vários trabalhos confirmam esse sinergismo (Indira \& Ramesh, 2011; Yongqin \& Tao, 2011; Sassi, 2012).

Assim, as chamadas redes NeuroFuzzy (RNFs) combinam a lógica Fuzzy com o aprendizado e as capacidades adaptativas das RNAs. Isso significa que se pode inicializar a RNA usando conhecimento apurado expresso com regras Fuzzy e então treiná-la usando dados numéricos. Após treinar a RNA, as regras podem ser avaliadas e editadas para sobrepor qualquer deficiência nos dados treinados.

A associação entre RNAs e a lógica Fuzzy tem sido frequentemente utilizada para resolver problemas não lineares e complexos. A integração dessas duas técnicas fornece para determinados tipos de problemas melhores resultados em comparação com as técnicas utilizadas isoladamente (Halgamude \& Glesner, 1994).

As RNFs têm sido utilizadas nas mais diversas áreas como em processos industriais (Er \& Zhou, 2008; Kebbe \& Danne, 2009; Muniraj \& Chandrasekar, 2011), na medicina (Gonzalez-Olivera et al., 2009), na previsão da taxa de câmbio brasileira (Coelho et al., 2008), na roteirização de veículos (Affonso et al., 2011), entre outras.

0 objetivo deste trabalho foi analisar o uso de uma RNA do tipo Multilayer Perceptrons (MLP) e de outra RNA do tipo Radial Basis Function (RBF) associadas à lógica Fuzzy (rede NeuroFuzzy) utilizando o modelo de inferência Mamdani (Mamdani \& Assilian, 1975) na previsão do tempo de ciclo de processos de injeção de polímeros. Foram utilizadas duas bases de dados: uma base de dados obtida através do acompanhamento de testes de funcionamento (tryouts) realizados em uma indústria de autopeças e outra base de dados denominada polímeros obtida do trabalho realizado por Galdamez \& Carpinetti (2004).

A continuidade deste trabalho está organizada da seguinte forma: na seção 2 discute-se o processamento de polímeros na indústria automotiva. Na seção 3 apresentam-se os princípios da lógica Fuzzy, as funções de pertinência e como funciona o mecanismo de inferência. Na seção 4 é apresentada teoria sobre as redes neurais artificiais e sobre as redes NeuroFuzzy. A seção 5 apresenta os experimentos computacionais e na seção 6 conclui-se o trabalho.

\section{Processamento de polímeros na indústria automotiva}

A produção de peças por injeção de polímeros (plásticos) é um dos processos de manufatura mais comuns, devido à alta capacidade de produção em larga escala e seus relativamente baixos custos de produção (He et al., 2001).

A aplicação de materiais poliméricos na produção de componentes do setor automotivo tende a crescer, principalmente para aplicação em peças de acabamento, como painel de instrumentos, bancos, teto etc. Segundo Hemais (2003), essa tendência se justifica exatamente pela flexibilidade das propriedades encontradas nos materiais poliméricos.

A moldagem de peças por injeção segundo Eyerer et al. (2008) é um processo que possui vantagens como:

- curto processo produtivo (desde a matéria-prima até o produto acabado);

- pouco ou nenhum retrabalho, processos integráveis e totalmente automatizados;

- alta repetitibilidade do processo;

- baixo consumo de energia (em comparação com os metais, devido às baixas temperaturas de processamento);

- integração das etapas de desenvolvimento desde a ferramenta protótipo até a ferramenta definitiva.

Garantir a qualidade desse processo é uma tarefa complexa, devido à influência de um grande número de fatores que vão desde o conceito do produto até a construção da ferramenta de injeção. Dentre os fatores podem se destacar (Eyerer et al., 2008):

- mão de obra (motivação, qualificação, flexibilidade, experiência, confiabilidade);

- máquina injetora (desempenho, precisão, segurança, nível de automação);

- ferramenta (sistema de aquecimento, projeto das partes móveis, acabamento); 
- matéria-prima (a seleção adequada, pureza, reciclabilidade);

- periféricos: controladores de temperatura, manipuladores automatizados;

- local de trabalho (segurança, meio ambiente, organização, limpeza).

Em um produto automotivo, como por exemplo uma cobertura de pára-choque injetada em polipropileno (PP), uma possível lista de propriedades desejadas, de acordo com Ryper (1996), seria: resistência à ação de intemperismo (sol, chuva, neve etc.), estabilidade dimensional, boa aderência e recobrimento da camada de tinta, resistência à corrosão por agentes químicos.

A Figura 1 mostra outros fatores envolvidos na obtenção de produtos injetados que atendam aos requisitos de qualidade.

Definida a classe de material que atenda às especificações, a escolha final recobre sobre o material que apresenta o melhor desempenho, analisando-se toda a cadeia produtiva.

Eyerer et al. (2008) recomendam observar também esses fatores:

- preço do material por quilo;

- peso específico;

- fatores de mercado;

- tecnologia empregada;

- processabilidade e porcentagem de refugo.

0 próximo passo é estabelecer uma relação das características do produto que se deve atender e é importante ter em mente que não existe uma lista única de fatores mais relevantes, tais propriedades estão univocamente relacionadas à aplicação do

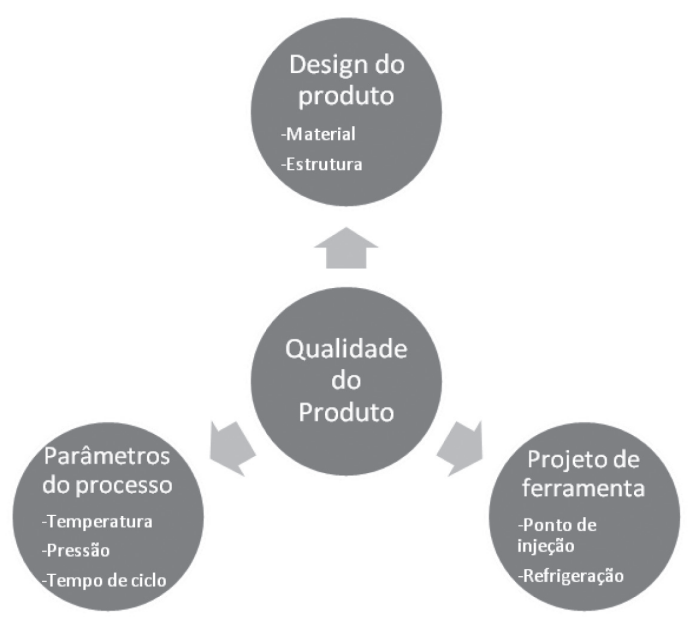

Figura 1. Fatores que influenciam na qualidade de peças injetadas. He et al. (2001). componente. Caso fosse definida uma lista única perder-se-ia exatamente o diferencial de materiais plásticos, ou seja, a sua flexibilidade.

Um dos critérios mais importantes para a seleção de materiais para produtos injetados é seu tempo de ciclo, pois ele determina a quantidade de peças que serão produzidas num determinado período e, portanto, seu custo de produção.

Uma vez escolhido o material que apresenta o melhor desempenho, o próximo passo é realizar teste em protótipos, à exaustão em todas as solicitações possíveis, sempre nas aplicações mais críticas, nos quais adaptações ou troca de materiais são desaconselháveis.

0 ganho com adaptações é infinitamente inferior a perdas em caso de erro de especificação. Após o término da construção das ferramentas de injeção são feitos testes de funcionamento (tryouts) para se determinar o melhor conjunto de parâmetros que fornece peças qualitativamente em ordem.

Os parâmetros iniciais escolhidos para esse tryouts, os chamados setup de processo, são determinados por experiência, ou similaridade com outros produtos. 0 processo de injeção é altamente complexo, sendo muito difícil de estabelecer o melhor setup na primeira tentativa. Dessa forma, o ajuste de processo é realizado através de tentativa e erro, o que torna o processo custoso.

0 processo de injeção de polímeros apresenta três etapas principais (Agassant et al., 1991): carregamento, injeção e resfriamento. $\mathrm{Na}$ fase de carregamento o polímero é alimentado através de um funil de alimentação e depois, devido ao atrito com as hélices da extrusora e da transferência de calor das resistências elétricas, este é fundido (Figura 2).

$\mathrm{Na}$ fase de injeção o material é empurrado para dentro da cavidade do molde a alta pressão e finalmente na fase de resfriamento o polímero troca calor com o molde de injeção até retornar à fase sólida. Os principais parâmetros do processo são apresentados na Tabela 1 (Billmeyer, 1971).

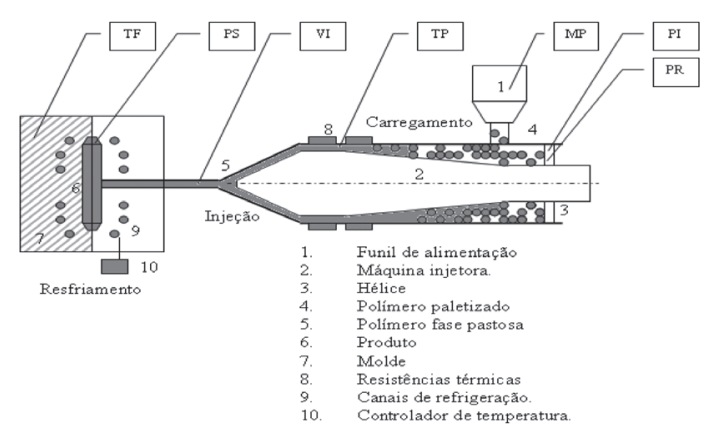

Figura 2. Processo de injeção de polímeros. 
Deve-se considerar também que os polímeros, assim como outros fluidos complexos, não obedecem à lei de Newton da viscosidade para a tensão de cisalhamento $\tau$, conforme a Equação 1:

$$
\tau=\eta \dot{\gamma}
$$

Esses fluidos exibem uma viscosidade $\eta$ decrescente com a taxa de $\gamma$. Yamaguchi (2008) refere-se a esse comportamento como viscoelástico. Nos polímeros a viscosidade aparente é uma função decrescente da taxa de cisalhamento. A mais comum expressão para 0 comportamento pseudoplástico é a lei da Potência ou modelo de Otswald-de Waele, conforme a Equação 2:

$$
\eta(\dot{\gamma})=K \dot{\gamma}^{n-1}
$$

A base de dados mostrada na Tabela 2 foi obtida através de acompanhamento de tryouts realizados em uma indústria de autopeças por especialistas na produção de autopeças injetadas.

0 ajuste de parâmetros de processo não seguiu nenhum planejamento de experimento predefinido, cada uma das peças partiu de parâmetros iniciais (setup de processo) diferentes, onde os parâmetros foram ajustados até que o processo produzisse peças qualitativamente em ordem. Os valores dos parâmetros de processo e produto obtidos nos tryouts após a estabilidade do processo ter sido alcançada são mostrados na Tabela 2.

Tabela 1. Variáveis do processo de injeção.

\begin{tabular}{ccc}
\hline $\mathrm{y}$ & Tempo de ciclo & $\mathrm{CL}$ \\
\hline $\mathrm{x}_{1}$ & Viscosidade & $\mathrm{MP}$ \\
$\mathrm{x}_{2}$ & Peso & $\mathrm{PS}$ \\
$\mathrm{x}_{3}$ & Temperatura do polímero & $\mathrm{TP}$ \\
$\mathrm{x}_{4}$ & Temperatura da ferramenta & $\mathrm{TF}$ \\
$\mathrm{x}_{5}$ & Pressão de injeção & $\mathrm{Pl}$ \\
$\mathrm{x}_{6}$ & Pressão de recalque & $\mathrm{PR}$ \\
$\mathrm{x}_{7}$ & Velocidade de injeção & $\mathrm{VL}$ \\
\hline
\end{tabular}

\section{Princípios da lógica Fuzzy}

He et al. (2001) mostram que a produção de peças por injeção de polímeros é um processo onde a modelação matemática direta é inviável, devido ao elevado número de variáveis e fenômenos físicos envolvidos.

Como é típico em processos industriais existem incertezas envolvidas na obtenção e controle das variáveis de processo, pois os parâmetros devem ser de fácil obtenção e controláveis para que possam ser utilizados no ambiente fabril.

Conforme Agassant et al. (1991), a análise do fenômeno pelos métodos clássicos é complexa devido aos seguintes fatores: os moldes são frequentemente de forma complexa, o fluxo acontece em regime não permanente num tempo de injeção de alguns segundos, os efeitos térmicos são relevantes e os polímeros apresentam reologia complexa.

Problemas desse tipo requerem a determinação de um mecanismo de inferência que represente de forma satisfatória a dinâmica do processo, ou seja, como as variáveis de entrada relacionam-se entre si, quais são suas saídas, e os correspondentes erros associados.

A exatidão do modelo é diretamente proporcional à aderência entre a dinâmica real do sistema e o mecanismo de inferência proposto para representá-la. Por esse motivo escolheu-se a lógica Fuzzy para a determinação desse mecanismo de inferência.

Conforme Russel (2007), para um dado elemento deve ser estabelecido se este pertence ou não a um conjunto. No mundo real o conceito de conjunto é utilizado de forma vaga.

Portanto, para uma grande gama de fenômenos físicos fica difícil estabelecer claramente se um elemento pertence ou não a um determinado conjunto. Zadeh (1965) propôs uma caracterização mais ampla introduzindo o conceito de lógica Fuzzy para representar essa classe de conjuntos.

Tabela 2. Valores das variáveis do processo de injeção de polímeros.

\begin{tabular}{ccccccccc}
\hline Try out & $\mathrm{MP}[\mathrm{g} / 10 \mathrm{~min}]$ & $\mathrm{PS}[\mathrm{g}]$ & $\mathrm{TP}\left[{ }^{\circ} \mathrm{C}\right]$ & $\mathrm{TF}\left[{ }^{\circ} \mathrm{C}\right]$ & $\mathrm{Pl}[\mathrm{bar}]$ & $\mathrm{PR}[\mathrm{bar}]$ & $\mathrm{VL}[\mathrm{s}]$ & $\mathrm{CL}[\mathrm{s}]$ \\
\hline 1 & 7 & 174 & 227 & 70 & 58 & 30 & 15 & 70,00 \\
2 & 13 & 932 & 240 & 30 & 90 & 45 & 5 & 80,00 \\
3 & 7 & 174 & 232 & 70 & 55 & 30 & 15 & 70,00 \\
4 & 7 & 174 & 227 & 70 & 65 & 30 & 15 & 70,00 \\
5 & 2,2 & 174 & 238 & 70 & 58 & 30 & 18 & 70,00 \\
6 & 2,2 & 174 & 243 & 70 & 65 & 45 & 20 & 70,00 \\
7 & 13 & 630 & 220 & 30 & 100 & 50 & 3 & 66,00 \\
8 & 13 & 630 & 230 & 45 & 84 & 40 & 4 & 84,00 \\
9 & 13 & 630 & 240 & 30 & 93 & 55 & 3 & 94,00 \\
10 & 13 & 932 & 220 & 30 & 93 & 50 & 5 & 68,00 \\
11 & 7 & 174 & 227 & 70 & 55 & 30 & 15 & 70,00 \\
12 & 13 & 932 & 240 & 30 & 90 & 45 & 5 & 80,00 \\
\hline
\end{tabular}


A seguir são dadas algumas definições: um conjunto Fuzzy A em um universo $\mathrm{X}$ é definido através de uma função de pertinência, conforme as Equações 3 e 4:

$$
\begin{aligned}
& \mu_{A}(x): X \rightarrow[0,1] \\
& A=\left\{\mu_{A}(x) / x\right\} x \in X
\end{aligned}
$$

onde $\mu_{A}(x)$ indica quanto $x$ é compatível com o conjunto $A$. Quanto mais próximo da unidade, maior a "afinidade" do elemento ao conjunto. Objetos são normalmente descritos através de agrupamentos no mundo real.

Tomando $X=\left\{x_{1}, x_{2}, \ldots, x_{n}\right\}$, como um conjunto de objetos a ser classificados, e os correspondentes índices de $x_{i}$ são $x_{i 1}, x_{i 2} \ldots, x_{i m}$. A questão é classificar $x$ baseado nesses índices e em geral esse problema é tratado pela estatística descritiva.

As relações Fuzzy fornecem uma solução alternativa, como mostrada a seguir. Deve-se transformar os índices $\left(\mathrm{x}_{\mathrm{i} 1}, \mathrm{x}_{\mathrm{i} 2}, \ldots, \mathrm{x}_{\mathrm{im}}\right)$ e $\left(\mathrm{x}_{\mathrm{j} 1}, \mathrm{x}_{\mathrm{j} 2}, \ldots, \mathrm{x}_{\mathrm{jm}}\right)$ de $x_{i}$ e $x_{j}$ em um número único $d_{i j}$ o qual reflita 0 grau de similaridade de $x_{i} \operatorname{com} x_{j}$ (Wang et al., 2009). Esse processo é conhecido como escalonamento.

Existem várias formas de se realizar o escalonamento, no presente trabalho foi utilizada a distância euclidiana para o escalonamento, conforme a Equação 5:

$$
d_{i j}=1-c \sum_{k=1}^{m}\left|x_{i k}-x_{j k}\right|
$$

0 mecanismo de inferência é uma questão central na modelagem do problema e será apresentada neste trabalho a forma de construí-lo. 0 estabelecimento do mecanismo de inferência que simula um sistema envolve dois estágios (Carvalho et al., 2008):

- as premissas de todas as regras são comparadas com as entradas controladas para determinar quais regras se aplicam para determinada situação;

- as conclusões são estabelecidas usando as regras que foram determinadas.

Para representar os mecanismos de inferência nos conjuntos Fuzzy foi utilizado o conceito de relação Fuzzy, que generaliza o conceito de relações da teoria clássica dos conjuntos e representa o grau da associação entre elementos de dois ou mais conjuntos Fuzzy.

Formalmente, dados dois conjuntos Fuzzy $X$ e $Y$. Um subconjunto do produto cartesiano R: $X \mathrm{x}$ $Y \rightarrow[0,1]$, é chamado relação, para $(\mathrm{x}, \mathrm{y}) \in(X \times Y)$, $\mathrm{R}(\mathrm{x}, \mathrm{y}) \in[0,1]$.

Para conjuntos finitos pode-se escrever a relação $R$ de $X$ para $Y, x=\left\{x_{1}, x_{2}, \ldots, x_{n}\right\}, y=\left\{y_{1}, y_{2}, \ldots, y_{m}\right\}$, neste caso escrevendo $r_{i j}=R\left(x_{i}, y_{j}\right)_{i=1, \ldots, n / j=1, \ldots, m}$.
Uma variável é frequentemente descrita através de palavras em linguagem natural, ao invés de números. 0 conceito variável linguística tem uma função importante na representação do conhecimento impreciso humano. A composição de múltiplas variáveis linguísticas pode constituir uma descrição sistemática do conhecimento humano em determinada área do conhecimento.

Neste trabalho, foi utilizado o operador sugerido por Mamdani \& Assilian (1975), onde o valor verdade de uma composição $\Phi$ que são as regras do mecanismo de inferência foi calculado conforme a Equação 6:

$$
\begin{aligned}
& \Phi_{i j}(x, y)=T\left(R_{j}(x), \mu_{i}^{\alpha}(y)\right) \\
& R_{j}(x)=\mu_{x 1}^{\alpha 1}\left(x_{1}\right) \wedge \mu_{x 2}^{\alpha 2}\left(x_{2}\right) \wedge \ldots \wedge \mu_{n}^{\alpha n}\left(x_{n}\right)
\end{aligned}
$$

Uma relação Fuzzy pode ser expressa linguisticamente através de proposições semelhantes a: "a temperatura é baixa" ou "a temperatura não é baixa", onde "baixa" representa um rótulo linguístico definido por um número Fuzzy no universo do discurso da variável linguística "temperatura". Neste trabalho, utilizou-se como regra de inferência Fuzzy o modus ponens generalizado.

0 próximo passo é aplicar a semântica da lógica Fuzzy na injeção de polímeros. Isto se deu através da criação de funções de pertinência para cada um dos parâmetros de processo e produto envolvidos no sistema de injeção, bem como a saída desejada.

0 critério para definição da amplitude, centros e desvio-padrão de cada uma dessas funções de pertinência $\mu$ foi obtido através de consultas com especialistas, pesquisa à bibliografia especializada e análise dos fenômenos de transporte envolvidos no sistema.

Foram considerados três rótulos linguísticos para as funções de pertinência: baixo, médio e alto.

Como é característica dos sistemas modelados através da lógica Fuzzy, a escolha dos parâmetros das funções de pertinência é arbitrária, entretanto determinará fortemente o sucesso ou insucesso da modelação. Conforme apresentado inicialmente, foram exploradas as principais características das funções de pertinência, bem como quais funções foram estabelecidas para o caso em estudo.

A função que melhor representa os fenômenos presentes no sistema estudado é a gaussiana (Passino \& Yurkovich, 1998), conforme a Equação 7:

$$
\mu^{\alpha}(x)=e^{-\frac{1}{\sigma}\left(x-c_{\alpha}\right)^{2}}
$$

onde:

- ca: centro da função

- $\sigma \alpha$ : desvio padrão

- $\alpha$ : rótulos linguísticos 
Para modelar o grau de pertinência da variável temperatura do polímero (TP) utilizaram-se três rótulos linguísticos: $\mathrm{a} 1-=$ baixa, a 0 = média e a 1 = alta; os respectivos valores para os centros das funções são: $\mathrm{c}-1=227{ }^{\circ} \mathrm{C} ; \mathrm{c} 0=235^{\circ} \mathrm{C}$ e c $1=240{ }^{\circ} \mathrm{C}$.

As Figuras 3 e 4 apresentam as funções de pertinência propostas para a temperatura da ferramenta (TF) e para a temperatura do polímero (TP).

A complexidade do produto possui um papel importante na determinação dos parâmetros do processo de injeção, e precisa ser levada em consideração para definição do modelo. Foram selecionados o peso da peça PS e a fluidez do material MP como as variáveis que representam a influência das características do produto que têm impacto nos valores do tempo de ciclo.

\subsection{Base de regras}

A norma $\mathrm{T}$ foi escolhida para operar sobre as funções de pertinência para obtenção das regras do mecanismo de inferência. 0 nível de ativação das regras é obtido aplicando-se uma norma $\mathrm{T}$ aos elementos da classe de treinamento. Foi adotado para essa norma o operador min. proposto por Carvalho et al. (2008).

A aplicação da lógica Fuzzy para previsão ou monitoramento, em um determinado sistema, é

\section{Função de Pertinência: TF}

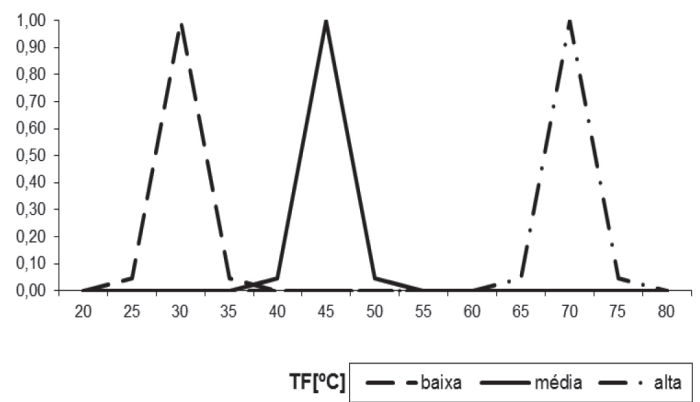

Figura 3. Função de pertinência da temperatura da ferramenta.

\section{Função de Pertinência: TP}

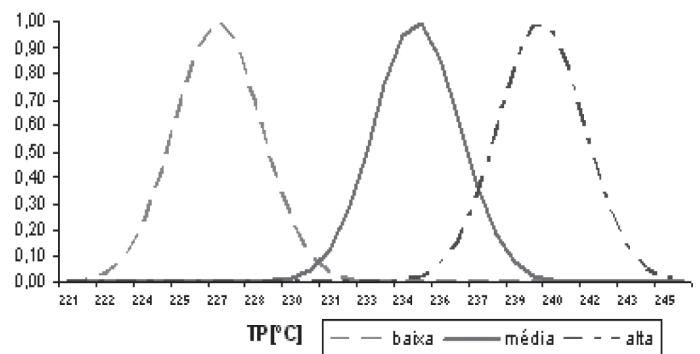

Figura 4. Função de pertinência da temperatura do polímero. caracterizada pela utilização de um mecanismo de inferência baseado em regras preestabelecidas. Essa característica gera a necessidade de que os eventos apresentados na entrada devem necessariamente se classificar em uma dessas regras. Assim, a base de dados de treinamento deve abranger todos os estados possíveis do sistema.

Entretanto, com a utilização da RNF o número de exemplos de treinamento pode ser reduzido em função da capacidade de aprendizado das RNAs tornando sua aplicação viável para casos onde o número de exemplos de treinamento é restrito. $\mathrm{Na}$ Tabela 3 é apresentada a base de regras para a fase de aprendizado, onde: rótulo linguístico "baixo" igual a -1 , rótulo linguístico "médio" igual a 0 e rótulo linguístico "alto" igual a 1.

A separação da base de dados entre dados de aprendizado e dados de generalização foi randômica, sendo aproximadamente $80 \%$ da base de dados para a fase de aprendizagem e $20 \%$ para a fase de generalização. Foram estabelecidas segundo os mesmos critérios as regras de inferência da fase de validação do modelo (Tabela 4).

0 critério utilizado para o classificação das regras de inferência foi a distância euclidiana.

\section{Conceitos básicos de redes neurais artificiais e de redes NeuroFuzzy}

As redes reurais artificiais (RNAs) são modelos inspirados na estrutura do cérebro tendo como objetivo simular o comportamento humano em processos como: aprendizagem, associação, generalização e abstração. Nessas redes, o aprendizado se dá através

Tabela 3. Base de regras da fase de aprendizado.

\begin{tabular}{cccccccccc}
\hline Regra & $\begin{array}{c}\text { Try } \\
\text { out }\end{array}$ & $\mu_{\mathrm{MF}}$ & $\mu_{\mathrm{PW}}$ & $\mu_{\mathrm{PT}}$ & $\mu_{\mathrm{MT}}$ & $\mu_{\mathrm{IP}}$ & $\mu_{\mathrm{RP}}$ & $\mu_{\mathrm{VL}}$ & $\mu_{\mathrm{CL}}$ \\
\hline F1 & 1 & 0 & -1 & -1 & 1 & -1 & -1 & 0 & 0 \\
F2 & 2 & 1 & 1 & 1 & -1 & 1 & 1 & -1 & 1 \\
F3 & 3 & 0 & -1 & 0 & 1 & -1 & -1 & 0 & 0 \\
F4 & 5 & -1 & -1 & 1 & 1 & -1 & -1 & 1 & 0 \\
F5 & 7 & 1 & 0 & -1 & -1 & 1 & 1 & -1 & -1 \\
F6 & 8 & 1 & 0 & 0 & 0 & 0 & 0 & -1 & 1 \\
F7 & 9 & 1 & 0 & 1 & -1 & 1 & 1 & -1 & 1 \\
F8 & 10 & 1 & 1 & -1 & -1 & 1 & 1 & -1 & 1 \\
\hline
\end{tabular}

Tabela 4. Base de regras da fase de generalização.

\begin{tabular}{cccccccccc}
\hline Regra & $\begin{array}{l}\text { Try } \\
\text { out }\end{array}$ & $\mu_{\mathrm{MF}}$ & $\mu_{\mathrm{PW}}$ & $\mu_{\mathrm{PT}}$ & $\mu_{\mathrm{MT}}$ & $\mu_{\mathrm{IP}}$ & $\mu_{\mathrm{RP}}$ & $\mu_{\mathrm{VL}}$ & $\mu_{\mathrm{CL}}$ \\
\hline F1 & 11 & 0 & -1 & -1 & 1 & -1 & -1 & 0 & 0 \\
F2 & 12 & 1 & 1 & 1 & -1 & 1 & 1 & -1 & 1 \\
F3 & 4 & 0 & -1 & 0 & 1 & -1 & -1 & 0 & 0 \\
F4 & 6 & -1 & -1 & 1 & 1 & -1 & -1 & 1 & 0 \\
\hline
\end{tabular}


de um conjunto de unidades simples de processamento chamados de neurônios artificiais ou nós.

Uma RNA do tipo Multilayer Perceptron (MLP) consiste de um conjunto de unidades (nós ou neurônios) que constituem a camada de entrada, uma ou mais camadas ocultas e uma camada de saída onde o sinal de entrada se propaga pela RNA camada por camada (Haykin, 2001).

0 algoritmo de treinamento usado na MLP é o error backpropagation (Haykin, 2001), que funciona da seguinte forma: apresenta-se um padrão à camada de entrada da rede, esse padrão é processado camada por camada até que a camada de saída forneça a resposta processada, $f_{\text {MLP }}$, como mostra a Equação 8:

$f_{M L P}(x)=\phi\left(\sum_{1}^{N o n} v_{l} \cdot \phi\left(\sum w_{l j} x_{l}+b_{l 0}\right)+b_{0}\right)$

onde $v_{1}$ e $w_{1 \mathrm{j}}$ são pesos sinápticos; $b_{10}$ e $b_{0}$ são os biases; e $\varphi$ a função de ativação, comumente especificada como sendo a função sigmóide.

0 princípio desse algoritmo para cálculo dos erros nas camadas intermediárias é o seu cálculo por retroalimentação, possibilitando dessa forma 0 ajuste dos pesos proporcionalmente aos valores das conexões entre camadas.

A MLP possui as seguintes características (Haykin, 2001): função de ativação não linear (sigmoidal), uma ou mais camadas de neurônios ocultos e um alto grau de conectividade.

Já uma rede neural artificial do tipo Radial Basis Function (RBF) em sua forma mais básica envolve três camadas:

- camada de entrada formada por unidades sensoriais que conectam a rede ao seu ambiente;

- camada interna em que se aplica uma transformada não linear do espaço de entrada para o espaço oculto, sendo que na maioria das aplicações o espaço oculto é de alta dimensionalidade;

- e a camada de saída linear fornece a resposta da rede ao padrão (sinal) de ativação aplicada à camada de entrada (Haykin, 2001).

Foi utilizada para aproximação da superfície de resposta uma combinação de funções de base radial, para esse caso em particular as funções de Green, conforme a Equação 9:

$$
\begin{aligned}
& F(x)=\sum_{i=1}^{m} w_{i} G\left(x, c_{i}\right) \\
& G\left(x-c_{i}\right)=e^{\frac{-\left(x_{j}-c_{i}\right)^{2}}{\sigma}}
\end{aligned}
$$

0 algoritmo de aprendizado escolhido para essa aplicação foi o K-médias, de forma que os pesos sinápticos $w$, e os centros das funções de base $c_{i}$ são recalculados, a partir dos parâmetros de aprendizagem $\eta_{1}$ e $\eta_{2}$, de forma minimizar o erro $\varepsilon$ conforme as Equações 10 e 11:

$$
\begin{aligned}
& w(n+1)=w(n)+\eta_{1} \sum \varepsilon(n) G_{i j}\left(x_{j}-c_{i}\right) \\
& c_{k}(n+1)=c_{k}(n)+\eta_{2}\left(x_{j}-c_{i}\right)
\end{aligned}
$$

Segundo Haykin (2001), a RBF e a MLP são aproximadores universais de funções, porém existem diferenças importantes entre elas: uma RBF possui sempre uma única camada oculta enquanto a MLP pode ter várias, a MLP constrói aproximações globais de um mapeamento de entrada-saída não linear. Por outro lado, a RBF utiliza não linearidades locais para o mapeamento da entrada e saída não lineares.

Para Rutkowski (2008) as redes NeuroFuzzy (RNFs) combinam RNAs com lógica Fuzzy resultando em sistemas com boa capacidade de aprender e de se adaptar às necessidades para a resolução de problemas do mundo real, ideais para aplicações como: identificação, predição, classificação e controle.

Segundo Nauck et al. (1996), o termo rede NeuroFuzzy é usado para sistemas que apresentam as seguintes propriedades:

- baseados em lógica Fuzzy e treinados por um algoritmo de aprendizado derivado de uma das RNAs;

- o processo de aprendizado opera em informações locais e causa apenas modificações locais no fundamento da rede NeuroFuzzy;

- possuem três camadas onde a primeira camada representa as variáveis de entrada, a camada do meio (escondida) representa as regras de inferência e a terceira camada representa as variáveis de saída (Figura 5);

- podem ser sempre interpretadas como um mecanismo de inferência - sendo que nem todos os modelos especificam procedimentos de aprendizado para criação de regras Fuzzy;

- o processo de aprendizado de uma RNF transforma as propriedades semânticas de um sistema Fuzzy em um conjunto de descrições - isso resulta em restrições que podem tornar-se modificações aplicáveis aos parâmetros do sistema, entretanto, nem todas as abordagens em uma rede Neuro Fuzzy possuem essa propriedade;

- aproximam uma função n-dimensional que é parcialmente definida pelo treinamento dos dados.

\section{Experimentos computacionais}

Os experimentos computacionais foram realizados em duas fases: 


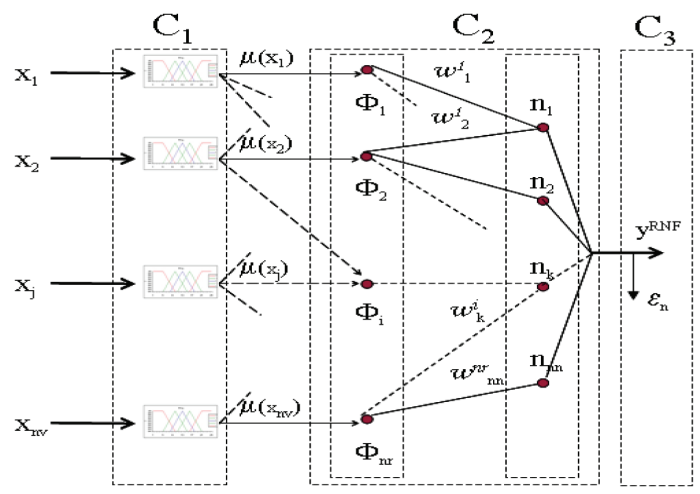

Figura 5. Arquitetura geral de uma RNF.

- na primeira fase comparou-se o resultado gerado pela RNF do tipo RBF com a RNF do tipo MLP. Essa comparação foi realizada utilizando a base de dados obtida através do acompanhamento de tryouts realizados em uma indústria de autopeças (Tabelas 1 e 2);

- na segunda fase deu-se a continuidade dos experimentos computacionais ao aplicar a RNF do tipo MLP na base de dados denominada polímeros, obtida do trabalho de Galdamez \& Carpinetti (2004) para validação.

Com relação às duas bases testadas (indústria de autopeças e polímeros) é importante destacar que se trata de bases de dados de naturezas distintas, pois no delineamento de experimentos via planejamento fatorial, no caso da base polímeros é necessário que as variáveis de entrada assumam valores predeterminados e que os experimentos sejam repetidos para que a superfície de resposta entrada-saída seja estabelecida. Por outro lado, na base da indústria de autopeças os exemplos são coletados randomicamente.

A plataforma de hardware utilizada nos experimentos foi um Pentium Dual Core com 2.4 MHZ, $512 \mathrm{MB}$ de memória RAM e $40 \mathrm{~GB}$ de disco rígido. 0 software utilizado para o experimento com a MLP foi o SCILAB 5.1 (http://www.scilab.org/products/scilab/ download), e para a RBF o algoritmo foi escrito pelos autores deste trabalho.

Os algoritmos desenvolvidos pelos autores do trabalho (totalmente no caso da rede RBF e parcialmente no caso da MLP) possibilitaram a análise das funções de ativação utilizadas e demais parâmetros de programação como: coeficiente de aprendizado, número de iterações, valor do erro absoluto, centro das funções gaussianas, entre outros.

\subsection{Experimento realizado com a rede NeuroFuzzy RBF (primeira fase)}

As RNAs possuem parâmetros de funcionamento que devem ser escolhidos para buscar melhor desempenho. No caso da RBF os parâmetros são: números de funções de base radial, número de neurônios, coeficientes de aprendizado e critérios de parada.

A RBF utilizada possui os seguintes parâmetros: número de neurônios de entrada igual a 8 , número de funções de base radial igual a 4, taxa de aprendizado constante: $\eta 1=1 \times 10^{-6}, \eta_{2=} 9.5 \times 10^{-4}$; critério de parada igual a erro médio absoluto $\varepsilon=0,65$ e número máximo de iterações ou épocas igual a 50. 0 número de funções de base radial e coeficientes de aprendizagem foram obtidos através de experimentação. Esses parâmetros foram ajustados até que os melhores resultados fossem encontrados.

A inferência do tipo Mamdani consiste em conectar os antecedentes e o consequente das regras usando normas $\mathrm{T}$ (seção 3.1, geralmente do tipo mínimo ou produto). Para este trabalho foi utilizado o sistema do tipo Mamdani levando-se em conta os pesos de cada regra $w$.

Na RNF do tipo RBF proposta neste trabalho os atributos são "fuzzyficados" através de funções de pertinência, conforme exemplificado nas Figuras 3 e 4.

Por meio do mecanismo de inferência estabelecido para o processo de injeção foram determinados os valores das regras $\Phi_{\mathrm{j}}$ conforme Equação 6, que servem de entrada para a primeira camada da RNF do tipo RBF.

A Figura 5 ilustra a arquitetura geral de uma RNF em que camada $C_{1}$ representa os valores crisp de entradas da rede, e a saída dessa camada corresponde aos valores das funções de pertinência. Na camada $C_{2}$ a rede é treinada a partir de um conjunto de regras de inferência.

Resende (2005) afirma que os pesos sinápticos da rede e os centros das funções de base são determinados de forma a se atingir os valores do target $\mathrm{y}$, os quais são os próprios valores do tempo de ciclo $\mathrm{CL}$ obtidos experimentalmente. $A$ camada $\mathrm{C}_{3}$ apresenta os valores de saída do sistema, correspondendo aos valores previstos pela RNF para a superfície de resposta.

Os 8 tryouts do grupo de treinamento da base de dados que foram inseridos na RNF do tipo RBF correspondem ao "professor" que mostra à rede qual padrão a rede deve seguir; baseado nesse padrão a rede aprendeu gerando uma matriz de pesos sinápticos. A seguir os dados do grupo de generalização (4 tryouts) são carregados no programa de forma a seguir o padrão estabelecido pela rede através dos pesos sinápticos.

A Figura 6 mostra a resposta da RNF do tipo RBF na fase de aprendizado e generalização em comparação com os dados reais, considerando como variável de saída o tempo de ciclo do processo de injeção. 
Como se pode observar na Figura 6 existe uma boa aderência entre os resultados da RNF do tipo RBF (RBF) e os dados experimentais (exp.), tanto para a fase de aprendizado quanto para a fase de generalização (a partir do $9^{\circ}$ tryout), indicando boa capacidade da RNF RBF para o modelamento do processo de injeção. Na Figura 7 é apresentado o valor do erro médio absoluto $(\varepsilon)$.

Uma característica importante no comportamento da RNF do tipo RBF é sua rápida convergência, pois com apenas 46 iterações (épocas) o ponto de corte foi atingido.

Para a RNF do tipo RBF o erro médio absoluto foi o critério para a avaliação de desempenho. Pode-se observar na Figura 7 que o desempenho melhorou com o aumento do número de iterações (épocas) caracterizando um melhor aprendizado da rede.

\subsection{Experimento realizado com a rede NeuroFuzzy MLP (primeira fase)}

Foi utilizado na RNF do tipo MLP o mesmo mecanismo de inferência discutido anteriormente para a RNF do tipo RBF. A diferença se deu na forma como as redes realizam o aprendizado e nas suas arquiteturas básicas. A seguir são apresentados os parâmetros utilizados para construção da RNF do

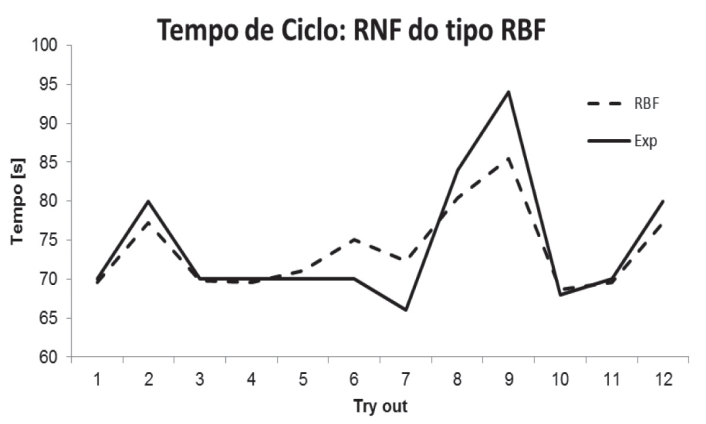

Figura 6. Resposta da RNF do tipo RBF na fase de aprendizado e generalização.

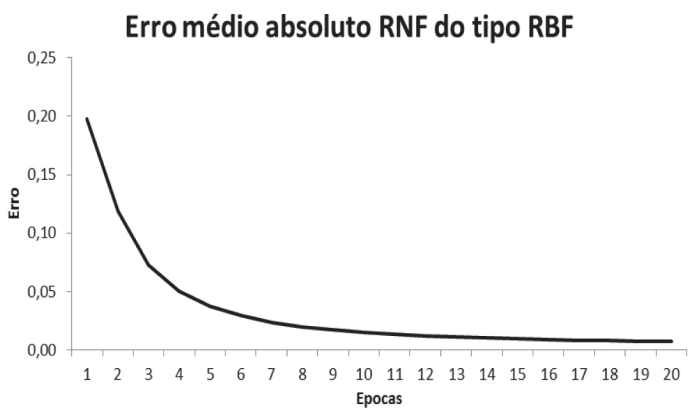

Figura 7. Erro médio absoluto da RNF do tipo RBF. tipo MLP: número de neurônios de entrada igual a 8 , número de camadas igual a 3 , taxa de aprendizado constante $\eta_{1=1,} \eta_{2}=0$, critério de parada igual ao erro médio absoluto $\varepsilon=0,85$ e número máximo de iterações ou épocas igual a 500 .

Para esse experimento a base de dados também foi separada em dois grupos: aprendizado e generalização. Posteriormente, a base de dados de aprendizado foi apresentada à rede para determinar o valor dos pesos sinápticos. No caso da RNF do tipo MLP foi necessária a criação de uma hipermatriz de dimensões $\operatorname{dim}\left[\mathrm{W}_{\text {MLP }}\right]=48$ posições para o mapeamento da superfície de resposta.

Uma vez realizado o aprendizado, os dados de generalização foram inseridos na RNF do tipo MLP seguindo o padrão estabelecido pelos pesos sinápticos gerando a superfície de resposta da rede (MLP) comparada aos dados experimentais (exp), conforme a Figura 8.

Também para a RNF do tipo MLP o erro médio absoluto foi o critério para a avaliação de desempenho. Pode-se observar na Figura 9 que o desempenho melhorou com o aumento do número de iterações (épocas) caracterizando um melhor aprendizado da rede.

Apesar do resultado semelhante apresentado pelas duas redes NeuroFuzzy utilizadas, elas apresentam diferenças estruturais com relação ao número de camadas, função de ativação, e a forma de mapeamento da superfície de resposta foi possivel verificar o desempenho diferenciado no aprendizado. A RNF do tipo RBF apresenta um aprendizado mais simples e rápido em relação à RNF do tipo MLP.

\subsection{Validação dos experimentos computacionais (segunda fase)}

0 objetivo foi validar as escolhas feitas com relação aos parâmetros das funções de pertinência e também com relação aos parâmetros da RNF do tipo MLP, ou seja, se a rede produz resultados satisfatórios quando utilizada outra base de dados. Como os resultados obtidos anteriormente foram semelhantes ao utilizar as duas RNFs optou-se, então, apenas por validar a RNF do tipo MLP.

Para tanto, foi utilizada a base de dados polímeros do trabalho de Galdamez \& Carpinetti (2004) construída através do planejamento fatorial $2^{k-p}$. Nesse caso, os valores de entrada foram definidos por meio de planejamento prévio, de forma a se obter resultados estatisticamente significativos. Conforme preconizados pelas técnicas de delineamento de experimentos foram levantados os fatores de controle que mais afetam a qualidade, conforme mostrado na Tabela 5. 
As variáveis de resposta que melhor definem a qualidade final do produto são classificadas em três grupos: desvios dimensionais, falha/rebarba e defeitos de superfície, pois são as que mais ocorrem e representam as maiores perdas econômicas no processo de fabricação. Os resultados são apresentados na Tabela 6.

No projeto experimental, para avaliar a falha e a rebarba do componente plástico foi construída uma

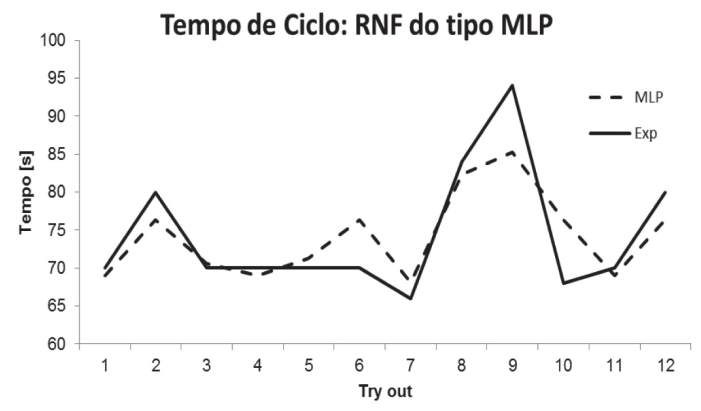

Figura 8. Superfície de resposta da RNF do tipo MLP.

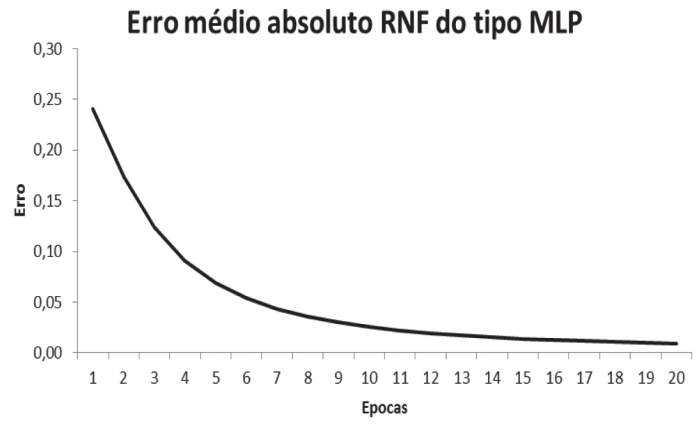

Figura 9. Erro absoluto médio da rede MLP. escala variando entre a nota -10 (falha) e a nota +10 (rebarba). Para os problemas de deformação e erro dimensional, a escala varia de 0 a 10 , sendo que a nota 10 é dada quando o produto apresenta deformação ou erro dimensional e 0 quando o componente plástico é aprovado pelo controle de qualidade. Para se determinar uma saída unidimensional foi utilizado o parâmetro mostrado na Equação 12:

$$
Y=(\text { Fal } / \text { Reb. })^{2}+(\text { Def. })^{2}+(\text { Dim })^{2}
$$

onde Y é uma variável adimensional que representa o nível de qualidade da peça injetada.

As análises de variância realizadas por Galdamez \& Carpinetti (2004) demonstraram que os parâmetros que têm maior influência no processo de injeção são a temperatura do polímero TP e a pressão de injeção Pl. A partir dos resultados obtidos, aplicando o método dos mínimos quadrados, foi construído um polinômio do segundo grau com os coeficientes mostrados na Equação 13:

$$
\begin{aligned}
Y & =12,35-8,45 T_{P}-10,14 P_{i} \\
& -20,8 T_{P}^{2}+15,2 P_{i}^{2}+42,80 T_{P} P_{i}
\end{aligned}
$$

A base de dados das Tabelas 5 e 6 foi utilizada como exemplo de treinamento. A capacidade de aprendizado e generalização da RNF do tipo MLP foi verificada por meio da aderência dos dados experimentais com sua saída. Na Figura 10 é apresentado o resultado da RNF do tipo MLP (MLP) comparado com os dados experimentais (exp).

0 erro médio absoluto decresce com o número de iterações. Foi encontrado o valor de erro médio

\begin{tabular}{|c|c|c|c|c|c|c|}
\hline Try out & $\mathrm{Tl}$ & TR & $\mathrm{TF}$ & $\mathrm{TP}$ & $\mathrm{VL}$ & $\mathrm{Pl}$ \\
\hline 1 & 2 & 6 & industrial & $190^{\circ} / 170^{\circ} / 165^{\circ} / 160^{\circ}$ & 40 & 18 \\
\hline 2 & 10 & 6 & industrial & $190^{\circ} / 170^{\circ} / 165^{\circ} / 160^{\circ}$ & 100 & 18 \\
\hline 3 & 2 & 15 & industrial & $190^{\circ} / 170^{\circ} / 165^{\circ} / 160^{\circ}$ & 100 & 30 \\
\hline 4 & 10 & 15 & industrial & $190^{\circ} / 170^{\circ} / 165^{\circ} / 160^{\circ}$ & 40 & 30 \\
\hline 5 & 2 & 6 & industrial & $190^{\circ} / 170^{\circ} / 165^{\circ} / 160^{\circ}$ & 100 & 30 \\
\hline 6 & 10 & 6 & industrial & $190^{\circ} / 170^{\circ} / 165^{\circ} / 160^{\circ}$ & 40 & 30 \\
\hline 7 & 2 & 15 & industrial & $190^{\circ} / 170^{\circ} / 165^{\circ} / 160^{\circ}$ & 40 & 18 \\
\hline 8 & 10 & 15 & industrial & $190^{\circ} / 170^{\circ} / 165^{\circ} / 160^{\circ}$ & 100 & 18 \\
\hline 9 & 2 & 6 & normal & $190^{\circ} / 240^{\circ} / 230^{\circ} / 220^{\circ}$ & 40 & 30 \\
\hline 10 & 10 & 6 & normal & $190^{\circ} / 240^{\circ} / 230^{\circ} / 220^{\circ}$ & 100 & 30 \\
\hline 11 & 2 & 15 & normal & $190^{\circ} / 240^{\circ} / 230^{\circ} / 220^{\circ}$ & 100 & 18 \\
\hline 12 & 10 & 15 & normal & $190^{\circ} / 240^{\circ} / 230^{\circ} / 220^{\circ}$ & 40 & 18 \\
\hline 13 & 2 & 6 & normal & $190^{\circ} / 240^{\circ} / 230^{\circ} / 220^{\circ}$ & 100 & 18 \\
\hline 14 & 10 & 6 & normal & $190^{\circ} / 240^{\circ} / 230^{\circ} / 220^{\circ}$ & 40 & 18 \\
\hline 15 & 2 & 15 & normal & $190^{\circ} / 240^{\circ} / 230^{\circ} / 220^{\circ}$ & 40 & 30 \\
\hline 16 & 10 & 15 & normal & $190^{\circ} / 240^{\circ} / 230^{\circ} / 220^{\circ}$ & 100 & 30 \\
\hline
\end{tabular}
absoluto $\varepsilon=5.759$ para 20 iterações. Na Figura 11

Tabela 5. Base de dados de validação dos experimentos (Polímeros - parte 1).

Fonte: Galdamez \& Carpinetti (2004). 
Tabela 6. Base de dados de validação dos experimentos (Polímeros - parte 2).

\begin{tabular}{ccccc}
\hline Try out & Fal/Reb & Def. & Din & Qualidade \\
\hline 1 & -10 & 10 & 10 & 300 \\
2 & -10 & 10 & 10 & 300 \\
3 & 0,75 & 4,2 & 0,95 & 19 \\
4 & -6 & 7 & 6,4 & 126 \\
5 & 2 & 1,2 & 1 & 6 \\
6 & 0 & 1 & 1 & 2 \\
7 & -10 & 10 & 10 & 300 \\
8 & -10 & 10 & 10 & 300 \\
9 & 8 & 7,6 & 3,25 & 132 \\
10 & 7 & 3 & 1 & 59 \\
11 & 2,75 & 4,4 & 1 & 28 \\
12 & 0,75 & 5 & 1 & 27 \\
13 & 5 & 5,3 & 3,7 & 67 \\
14 & 2 & 1,6 & 1 & 8 \\
15 & 6,25 & 3,4 & 1 & 52 \\
16 & 8,5 & 3 & 1,9 & 85 \\
\hline Fonte: Gldamez \& & & & &
\end{tabular}

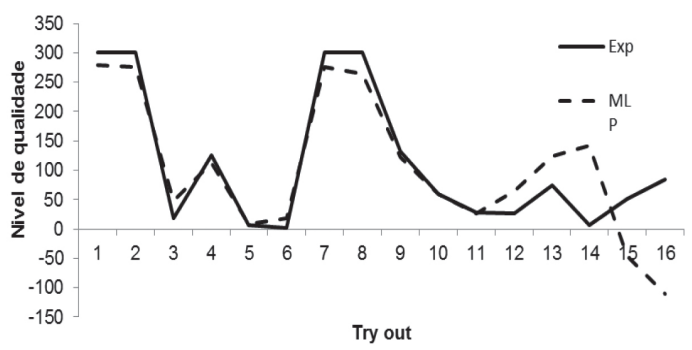

Figura 10. Resposta da RNF do tipo MLP na fase de aprendizado e generalização.

\section{Erro RNF do tipo MLP: Base Polímeros}

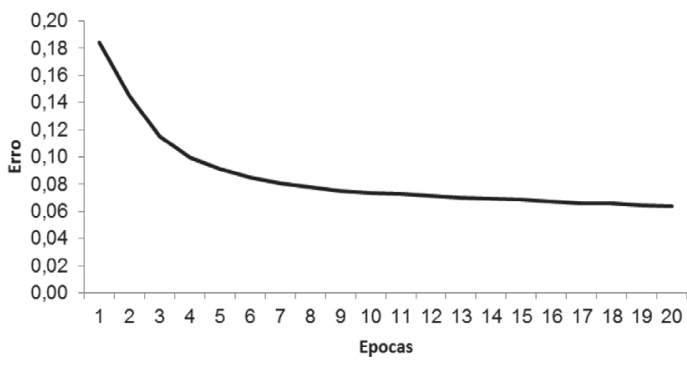

Figura 11. Erro médio absoluto da RNF do tipo MLP.

são apresentados os erros encontrados em função das iterações.

Os bons resultados obtidos encorajam a utilização de RNFs em problemas de processamento de polímeros.

\section{Conclusões}

0 uso de materiais poliméricos tem aumentado consideravelmente devido ao seu custo competitivo aliado a sua versatilidade. Com esse aumento surge a busca por novas tecnologias que auxiliem na seleção de materiais que apresentem melhor desempenho, tanto do ponto de vista econômico quanto em relação à economia de energia. Motivadas por esse cenário as técnicas de inteligência artificial são uma alternativa interessante para resolver problemas nessa área.

A aplicação da lógica Fuzzy mostrou-se uma ferramenta interessante para a captação e análise de incertezas relacionadas ao processo de injeção de polímeros, uma vez que esse processo possui características que dificultam a aplicação de um equacionamento direto, como por exemplo: caráter não linear, multifenômeno e fortemente dependente do especialista humano.

RNAs foram associadas à lógica Fuzzy numa abordagem denominada de rede NeuroFuzzy a fim de utilizar a capacidade de aprendizado e de generalização das RNAs. Foram criadas duas redes NeuroFuzzy: uma com a RNA do tipo MLP e a outra com a RNA do tipo RBF.

Por se tratar de um trabalho de aplicação foi necessário obter uma base de dados que representasse os processos de produção de peças por injeção de polímeros em ambientes fabris. Para a construção dessa base ocorreu um trabalho de campo, com acompanhamento de tryouts, bem como um intenso contato com os especialistas da área.

Com a base de dados estabelecida foram construídas funções de pertinência para as variáveis envolvidas no processo de injeção, baseadas no conhecimento extraído dos especialistas, bem como em consultas a manuais e normas técnicas, descritivos de matéria-prima e a bibliografia sobre o tema.

Um ponto crítico para a definição do mecanismo de inferência foi a determinação das regras de inferência, uma vez que foi necessário transformar informações verbais obtidas junto aos especialistas humanos em variáveis e conjuntos Fuzzy.

Para a realização dos experimentos computacionais optou-se pela estratégia de inferência de Mamdani. Determinou-se também como deveriam ser estruturadas as entradas: se acopladas através das regras de inferência ou diretamente pelas funções de pertinência. Optou-se pela técnica sugerida por Mamdani (Mamdani \& Assilian, 1975) com entradas acopladas.

Tanto a RNF do tipo RBF quanto a RNF do tipo MLP mostraram resultados aderentes aos dados extraídos das bases de dados podendo considerar que:

- as características de produto e processo podem ser corretamente caracterizadas através dos termos da lógica Fuzzy,

- pode ser estabelecido um mecanismo de inferência em peças injetadas com diferentes materiais $\mathrm{e}$ geometrias; 
- o uso das RNAs com a lógica Fuzzy para o tipo de problema considerado fornece um modelo com boa capacidade de aprendizado e generalização;

- os resultados apontam para uma potencial utilização de redes NeuroFuzzy tanto para predição de tempos de ciclo quanto para qualidade de peças injetadas;

- a análise comparativa entre as RNFs mostrou que a RNF RBF foi capaz de generalizar com maior precisão e com um número menor de iterações em comparação com a RNF MLP, apesar de no final dos experimentos os resultados serem muito semelhantes;

- os resultados obtidos também permitem identificar as melhores estratégias de setup para obtenção dos menores tempos de ciclo com potencial de otimização da utilização dos recursos energéticos e uma melhoria na qualidade do produto final.

Esses resultados encorajam a utilização de RNFs em problemas de processamento de polímeros.

Deve-se destacar que a geração de conhecimento confiável é uma tarefa notoriamente difícil, principalmente quando especialistas humanos não estão disponíveis. Portanto, sugere-se em trabalhos futuros a associação de outras técnicas da inteligência artificial às RNFs a fim de tentar diminuir a necessidade da utilização de especialistas na construção do modelo de inferência.

Os estudos aqui realizados não têm a pretensão de esgotar o assunto, pelo contrário, buscou-se realizar uma contribuição com a aplicação das RNFs. Sabe-se que existe uma clara demanda por estudos sistematizados no processamento de peças automotivas por meio de injeção de polímeros. Esse cenário oferece, portanto, amplo espaço para trabalhos de continuidade.

\section{Referências}

Affonso, C., Sassi, R. J., \& Pinto, R. P. (2011). Traffic flow breakdown prediction using feature reduction through rough-neuro fuzzy networks. In Proceedings of the International Joint Conference on Neural Networks, San Jose. http://dx.doi.org/10.1109/1JCNN.2011.6033462

Agassant, J. F., Avenas, P., Sergent, J., \& Carreau, P. J. (1991). Polymer Processing: principles and modeling. Continum Mechanics. Hanser Publishers.

Aguiar, D. C., \& Salomon, V. A. P. (2007). Avaliação da prevenção de falhas em processos utilizando métodos de tomada de decisão. Produção, 17(3), 502-519. http:// dx.doi.org/10.1590/S0103-65132007000300008

Amatucci, M., \& Bernardes, R. C. (2009). Formação de competências para o desenvolvimento de produtos em subsidiárias brasileiras de montadoras de veículos. Produção, 19(2), 359-375. http://dx.doi.org/10.1590/ S0103-65132009000200011

Billmeyer, F. W. (1971). Textbook of polymer science. John Wiley \& Sons.

Bissoto, 1., \& Sydenstricker, T. H. (2006). Substituição de materiais poliméricos através de seletores de materiais e fornecedores: um estudo de caso na indústria automobilística. Polímeros, 16(3), 217-233. http:// dx.doi.org/10.1590/S0104-14282006000300011
Blazadonakis, E., \& Michalis, Z. (2008). Support Vector Machines and Neural Networks as Marker Selectors in Cancer Gene Analysis. Intelligent Techniques and Tools for Novel System Architectures. Berlin: Springer-Verlag Berlin Heidelberg.

Canevarollo, S. V. (2002). Ciência dos polímeros: um texto básico para tecnólogos e engenheiros. Artliber Editora.

Cao, L., Wang, J., Jiang, P., \& Jin, Q. (2011). Multiobjective intelligence optimal operation of PET polymerization. In Proceedings of the World Congress on Intelligent Control and Automation, Taipei. http://dx.doi.org/10.1109/ WCICA.2011.5970754

Carvalho, L. M. F., Nassar, S. M., Azevedo, F. M., Carvalho, H. J. T., Monteiro, L. L., \& Rech, C. M. Z. (2008). A neurofuzzy system to support in the diagnostic of epileptic events using different fuzzy arithmetical operations. Neuropsiquiatria, 79-83.

Coelho, L. S., Santos, A. A. P., \& Costa Junior, N. C. A. (2008). Can we forecast Brazilian exchange rates? Empirical evidences using computational intelligence and econometric models. Gestão \& Produção, 15(3), 635-647. http://dx.doi.org/10.1590/S0104-530X2008000300016

Contant, S., Lona, L. M. F., \& Calado V. M. A. (2004). Predição do Comportamento Térmico de Tubos Compósitos Através de Redes Neurais. Polímeros, 14(5), 295-300. http://dx.doi.org/10.1590/S0104-14282004000500004

Correia, T. C. V. D., Mello, J. C. C. B. S., \& Meza, L. A. (2011). Eficiência técnica das companhias aéreas brasileiras: um estudo com análise envoltória de dados e conjuntos nebulosos. Produção, 21(4), 676-683. http://dx.doi. org/10.1590/S0103-65132011005000049

Er, M. J., \& Zhou, Y. (2008). Dynamically Self-generated Fuzzy Neural Networks with Industry Applications. Soft Computing Applications in Industry Studies in Fuzziness and Soft Computing, 226, p. 47-68. http://dx.dovi. org/10.1007/978-3-540-77465-5_3

Eyerer, P., Hirth, T., \& Elsner, P. (2008). Polymer Engineering Technologien und Praxis. Berlin: Springer-Verlag.

Galdamez, E. V., \& Carpinetti, L. C. (2004). Application design of experiments in the injection molding process. Gestão \& Produção, 11(1), 121-134. http://dx.doi.org/10.1590/ S0104-530X2004000100011

Glauch, D., Rajadhyaksha, M., Buchmann, H. F., Martell, S. R., Schwab, H., \& Pilato, L. (1997). Injection molding techniques for epoxy molding compounds. In Proceedings of the Electrical Insulation Conference and Electrical Manufacturing \& Coil Winding Conferenc, Rosemont. http://dx.doi.org/10.1109/EEIC.1997.650955

Goldschmidt, R., \& Passos, E. (2005). Data Mining um guia prático. Conceitos, Técnicas, Ferramentas, Orientações e Aplicações. Rio de Janeiro: Campus.

Gonzalez-Olivera, M. A., Gallardo-Hernandez, A. G., Tang, Y., Revilla-Monsalve, M. C., \& 1slas-Andrade, S. (2009). A Continuous-Time Recurrent Neurofuzzy Network for Black-Box Modeling of Insulin Dynamics in Diabetic Type-1 Patients. Advances in Computational Intelligence, 61, 219-228.

Guarnieri, P., \& Hatakeyama, K. (2010). Formalização da logística de suprimentos: caso das montadoras e fornecedores da indústria automotiva Brasileira. Produção, 20(2), 186-199. http://dx.doi.org/10.1590/ S0103-65132010005000020

Halgamude, S. K., \& Glesner, M. (1994). Neural networks in designing Fuzzy systems for real world applications. Fuzzy Sets and Systems, 65, 1-12. 
Haykin, S. (2001). Neural Networks: a comprehensive foundation. New York: Willey \& Sons.

He, W., Zhang, F. Y., \& Liu, T. 1. (2001). Development of a Fuzzy Neuro System for Parameter Resetting of injection Molding. Journal of Manufacturing Science and Engineering, 110-118.

Hemais, C. A. (2003). Polímeros e a indústria automobilística. Polímeros, 13(2), 107-114.

Indira, S. U., \& Ramesh, A. C. (2011). Image segmentation using artificial neural network and genetic algorithm: a comparative analysis. In Proceedings of the International Conference on Process Automation, Control and Computing, Coimbatore. http://dx.doi.org/10.1109/ PACC.2011.5979059

Kebbe, D. A., \& Danne, C. (2009). On Adapting Neural Network to Cellular Manufacturing. Complex Systems and Self-organization Modelling. Berlin: Springer-Verlag.

Kovács, Z. L. (1996). Redes Neurais Artificiais: Fundamentos e Aplicações. Ed. Collegium Cognitio.

Mamdani, E. H., \& Assilian, S. (1975). An experiment in linguistic synthesis with a fuzzy logic controller. International Journal of Human-Computer Studies, 7, 1-13.

Medeiros, S., Mello, R., \& Campos Filho, P. (2007). Análise de projetos para unidades de conservação, usando lógica fuzzy. Produção, 17(2), 317-329. http://dx.doi. org/10.1590/S0103-65132007000200008

Muniraj, C., \& Chandrasekar, S. (2011). Adaptive neuro-fuzzy inference system for monitoring the surface condition of polymeric insulators using harmonic content. Generation, Transmission \& Distribution, 5(7), 751-759. http://dx.doi.org/10.1049/iet-gtd.2010.0383

Nauck, D., Klawonn, F., \& Kruse, R. (1996). Foundations of Neuro Fuzzy Systems. Willey \& Sons.

Ozturk, B., Gromala, P., Otto, C., Fischer, A., Jansen, K. M. B., \& Ernst, L. J. (2012). Characterization of adhesives and interface strength for automotive applications. In Proceedings of the International Conference on Thermal, Mechanical and Multi-Physics Simulation and Experiments in Microelectronics and Microsystems, Cascais. http:// dx.doi.org/10.1109/ESimE.2012.6191807

Passino, K. M., \& Yurkovich, S. (1998). Fuzzy Control. Addison Wesley Longman.

Resende, S. 0. (2005). Sistemas Inteligentes: fundamentos e aplicações. Manole.
Rutkowski, L. (2008). Computational Intelligence: methods and techniques. Berlin: Springer-Verlag.

Russel, B. (2007). Introdução a Filosofia Matemática. Rio de Janeiro: Jorge Zahar.

Ryper, R. (1996). Using Advanced material to improve automotive part life. In Proceedings of the Transmission and Driveline Symposium.

Salerno, M. S., Miranda, Z., Kamisaki, F. Y., \& Maluta, G. (2010). Alavancando pesquisa, desenvolvimento e inovação no setor de autopeças: análise e propostas a partir de survey e estudo qualitativo focado. Produção, 20(4), 565-575. http://dx.doi.org/10.1590/S0103-65132010005000059

Sassi, R. J. (2012). An hybrid architecture for clusters analysis: rough sets theory and self-organizing map artificial neural Network. Pesquisa Operacional, 32(1), 139-164. Http://dx.doi.org/10.1590/S0101-74382012005000001

Selau, L. P. R., \& Ribeiro, J. L. D. (2009). Methodology for the construction and choice of credit risk prediction models. Gestão \& Produção, 16(3), 398-413. http://dx.doi. org/10.1590/S0104-530X2009000300007

Steiner, M. T. A., Nievola, J. C., Soma, N. Y., Shimizu, T., \& Steiner Neto, P. J. (2007). Extração de regras de classificação a partir de redes neurais para auxílio à tomada de decisão na concessão de crédito bancário. Pesquisa Operacional, 27(3), 407-426. http://dx.doi. org/10.1590/S0101-74382007000300002

Wang, X., Ruan, D., \& Kerre, E. (2009). Mathematics of Fuzziness. Berlin: Springer-Verlag.

Yamaguchi, H. (2008). Engineering Fluid Mechanics. Springer Science Business Media.

Yongqin, L., \& Tao, L. (2011). Enterprise financial prediction analysis based on rough set and neural network. In Proceedings of the International Conference on E-Business and E-Government, Shanghai. http://dx.doi. org/10.1109/1CEBEG.2011.5882136

Zadeh, L. A. (1965). Fuzzy Sets. Information and Control, 8, 338-353.

\section{Agradecimentos}

Agradecemos à revista Produção e aos revisores anônimos pelas relevantes considerações.

\section{Application of neurofuzzy networks for the processing of automotive parts by polymer injection molding}

\section{Abstract}

The injection molding of automotive parts is a complex process due to the many non-linear and multivariable phenomena that occur simultaneously. Commercial software applications exist for modeling the parameters of polymer injection but can be prohibitively expensive. It is possible to identify these parameters analytically, but applying classical theories of transport phenomena requires accurate information about the injection machine, product geometry, and process parameters. However, neurofuzzy networks, which achieve a synergy by combining the learning capabilities of an artificial neural network with a fuzzy set's inference mechanism, have shown success in this field. The purpose of this paper was to use a multilayer perceptron artificial neural network and a radial basis function artificial neural network combined with fuzzy sets to produce an inference mechanism that could predict injection mold cycle times. The results confirmed neurofuzzy networks as an effective alternative to solving such problems.

Keywords

Automotive industry. Polymers. Injection mold. Neurofuzzy network. 